\title{
Abnormal spatial asymmetry of selective attention in ADHD
}

\author{
Edgar Chan, ${ }^{1}$ Jason B Mattingley, ${ }^{1}$ Cynthia Huang-Pollock, ${ }^{2}$ Therese English, ${ }^{3}$ \\ Robert Hester, ${ }^{1}$ Alasdair Vance, ${ }^{3}$ and Mark A. Bellgrove ${ }^{1}$ \\ ${ }^{1}$ The University of Queensland, School of Psychology and Queensland Brain Institute, Brisbane, Australia; \\ ${ }^{2}$ Department of Psychology, The Pennsylvania State University, USA; ${ }^{3}$ Academic Child Psychiatry Unit, \\ Department of Paediatrics, University of Melbourne, Royal Children's Hospital, Murdoch Children's \\ Research Institute, Melbourne, Australia
}

\begin{abstract}
Background: Evidence for a selective attention abnormality in children with attention deficit hyperactivity disorder (ADHD) has been hard to identify using conventional methods from cognitive science. This study tested whether the presence of selective attention abnormalities in ADHD may vary as a function of perceptual load and target lateralisation. Given evidence of right-hemisphere dysfunction in ADHD we predicted increased interference effects for right, but not left-sided target displays, particularly under low perceptual load. Method: Fourteen children with ADHD-C and 14 typically developing children were tested on a modified flanker task under low and high perceptual load. We also sought evidence for our hypothesis in a re-analysis of an independent data set (42 ADHD; 34 typically developing) in which load effects on selective attention in ADHD were previously examined (Huang-Pollock, Nigg, \& Carr, 2005). Results: As predicted, all children showed evidence of greater interference by flankers under low compared with high perceptual load conditions. Crucially, however, children with ADHD showed the greatest interference effect for right-sided target displays under low but not high perceptual load. In contrast, typically developing children showed the greatest interference for left-sided target displays. The magnitude of interference for right-sided targets was also positively correlated with ADHD symptom levels. Re-analysis of an independent data set (Huang-Pollock et al., 2005) further confirmed our findings. Conclusions: This study demonstrates that interference effects in children with ADHD and typically developing children are spatially asymmetrical but opposite in direction. The pattern of right-sided interference effects in children with ADHD suggests disruption within right hemisphere attentional networks in ADHD. Keywords: Selective attention, flanker interference, spatial bias, ADHD, spatial attention.
\end{abstract}

Attention deficit hyperactivity disorder (ADHD) is a neurodevelopmental disorder that affects around 4-6\% of children worldwide (Polanczyk \& Rohde, 2007). Although problems of 'distractibility' and 'inattention' are diagnostic for the disorder, objective methods from cognitive science have failed to identify the presence of any selective attention deficits compared to non-ADHD controls on classic paradigms including the Stroop and Eriksen Flanker tasks (Brodeur \& Pond, 2001; Huang-Pollock et al., 2005; Sergeant, Geurts, Huijbregts, Scheres, \& Oosterlaan, 2003). When deficits are reported, they tend to be of small effect size, requiring large samples for group differences to emerge (Johnson et al., 2008). Using a variant of the Eriksen flanker task (Eriksen \& Eriksen, 1974) we show that under conditions of low perceptual load interference effects in children with ADHD and typically developing children are spatially asymmetrical but opposite in direction.

Experimental work in cognitive science has identified a number of factors that influence selective attention. Across a series of studies Lavie and

Conflict of interest statement: No conflicts declared. colleagues (Lavie, 1995; Lavie, Hirst, de Fockert, \& Viding, 2004) examined the influence of perceptual load on the degree to which task-irrelevant stimuli cause interference within a Flanker task. In one version of the task, participants make a speeded response to a target stimulus ' $x$ ' or ' $z$ ' that appears along a central horizontal row, while ignoring a congruent or incongruent flanker that appears either above or below the target (Lavie et al., 2004) . The perceptual load of the task is manipulated by varying the number of non-target stimuli (none versus five non-targets) within the central display containing the target stimulus. The magnitude of interference (i.e., the interference effect) generated by the incongruent flankers is calculated as the difference in reaction time between congruent and incongruent conditions. According to Lavie's load theory of visual selection (Lavie, 1995), as display set size increases from one to six items, the flanker interference effect is expected to decrease. That is, when the perceptual demands of a task are relatively low, residual attentional capacity permits the automatic processing of irrelevant or distracting stimuli (i.e., late selection'; Duncan, 1980). By contrast, high perceptual processing demands exhaust available capacity and thus block processing of irrelevant 
stimuli at an earlier stage of perception (i.e., 'early selection'; Broadbent, 1958). Thus, low perceptual load conditions give rise to greater distractor interference effects than do high perceptual load conditions.

Recently, the influence of perceptual load on the interference effect was examined in children with ADHD (Huang-Pollock et al., 2005). In that study, a flanker paradigm was used and the perceptual load of the task was manipulated by varying the number of non-targets $(0,1,3$ or 5$)$ in separate trials. Children with ADHD performed comparably to typically developing children, showing similar response times and error rates under all levels of perceptual load. These findings are somewhat surprising given that ADHD is often thought to be associated with impaired selective attention or under-selectivity. Under these circumstances, one might have hypothesised impaired performance under both low and high perceptual load conditions in children with ADHD, relative to typically developing children. That this did not occur suggests that perceptual processing capacities in ADHD are comparable to those of controls, at least for the visual modality.

One possibility is that selective attention abnormalities in ADHD might be asymmetrical, being lateralised to either the left or right visual field. A number of studies of visual attention in ADHD have reported that children and adults are poorer at attending to, or slower to act upon, stimuli presented in the left versus the right visual field (Geeraerts, Lafosse, Vaes, Vandenbussche, \& Verfaillie, 2007; Sheppard, Bradshaw, Mattingley, \& Leeb, 1999; Voeller \& Heilman, 1988; but see Huang-Pollock \& Nigg, 2003). The phenomenon of left spatial inattention, or a rightward spatial bias, is also seen in patients with acquired damage to the right hemisphere (Driver \& Mattingley, 1998) and has prompted some to argue that ADHD might be a developmental form of neglect (Voeller \& Heilman, 1988; Heilman et al., 1991). Asymmetric attentional performance amongst children and adults with ADHD is consistent with a large body of structural and functional imaging work which has shown abnormalities in frontal, striatal and parietal areas of the right-hemisphere in ADHD (Booth et al., 2005; Sowell et al., 2003; Vance et al., 2007). We therefore asked whether distractor interference effects in children with ADHD were spatially asymmetrical. If children with ADHD display left-sided selective inattention (i.e., a rightward spatial bias), then interference effects associated with left-sided target displays should be significantly reduced compared to those associated with right-sided target displays, particularly under low perceptual load. Similar effects have already been reported in adult patients with acquired damage to the right hemisphere (Lavie \& Robertson, 2001). We further sought independent confirmation of this hypothesis via a re-analysis of the published data of Huang-Pollock et al. (2005). In that study the spatial position of the distractors was not analysed as a possible factor.

The current study therefore aimed to investigate whether manipulating perceptual load and the spatial location of targets would differentially influence the selective attention performance of children with ADHD compared with typically developing children. The perceptual load paradigm employed in the current study was a variant of the task described by Lavie and colleagues (2004). Participants were presented with a horizontal stimulus array. Under high perceptual load conditions, the array included one target and five non-targets. Under low perceptual load conditions, the target occurred alone. Targets occurred in left, central and right spatial locations. Distractor items always appeared centrally, above or below fixation, and were either congruent or incongruent with respect to the target.

We predicted that if selective attention abnormalities in ADHD are both spatially and load dependent, then children with ADHD should display larger interference effects when targets appear on the right and smaller interference effects when targets appear on the left, particularly under low perceptual load.

\section{Method}

\section{Participants}

Twenty-eight closely matched children (14 ADHD and 14 controls; age range 8-14) participated in the study. Children with ADHD were recruited through the Royal Children's Hospital ( $\mathrm{RCH})$ Melbourne, Australia, in accordance with the ethical guidelines of the $\mathrm{RCH}$ and the University of Melbourne. Typically developing children were recruited through schools of the Catholic Education System in Melbourne, Australia. Informed consent was appropriately obtained from all participant parents.

Children recruited into the ADHD group (12 males; Mean age $=11.7$ years, range $8-14$ years) had all undergone a full cognitive and behavioural assessment at the $\mathrm{RCH}$ within two years of the current study and met criteria for ADHD-combined type (ADHD-CT) according to DSM-IV criteria (American Psychiatric Association, 1994). Of the 14 children with ADHD, 12 had comorbid diagnoses as assessed by the DSM-IV (conduct disorder $(n=10)$, oppositional defiant $(n=2)$, anxiety/mood disorder $(n=7))$. Children were excluded if their performance on the Wide Range Achievement Test - Reading Subtest (WRAT-R) was 1.5 SDs or more below normative values, estimated full-scale IQ $\leq 80$ on the WISC-IV, or if they had previously been diagnosed with impaired sensorimotor skills, learning disabilities or symptoms of psychosis. Within one week of participating in the current study, parents were required to complete the Parent Conners' ADHD Rating ScaleRevised: Long Version (CPRS-R:L; Conners, 1997), and children recruited into the ADHD group were required to have a Global Index T-scores > 65 . 
Table 1 Description of the group demographics and scores on standardised achievement and behavioural measures

\begin{tabular}{lccr}
\hline & Control $(n=14)$ & ADHD $(n=14)$ & \\
& $\mathrm{M}(\mathrm{SD})$ & $74.71(7.16)$ & $p$ value \\
\hline Conners' ADHD Index T-score & $45.36(5.08)$ & $80.50(7.41)$ & $<.001$ \\
Conners' Global Index T-score & $49.14(5.80)$ & $73.07(7.45)$ & $<.001$ \\
DSM-IV Inattentive T-score & $45.21(5.41)$ & $80.93(7.55)$ & $<.001$ \\
DSM-IV Hyperactive/Impulsive T-score & $50.14(6.34)$ & $79.21(7.94)$ & $<.001$ \\
DSM-IV Total T-score & $48.64(7.25)$ & $97.79(11.61)$ & $<.001$ \\
WRAT Reading score & $104.79(14.01)$ & $92.43(15.72)$ & $>.05$ \\
WRAT Spelling score & $105.79(12.71)$ & $100.36(8.96)$ & $=.02$ \\
Full-scale IQ & $106.86(8.37)$ & $11.67(2.16)$ & $>.05$ \\
Age in years & $11.02(1.14)$ & $>.05$ \\
\hline
\end{tabular}

The values shown are mean scores with standard deviations in parenthesis. Abbreviations: DSM-IV = Diagnostic and Statistical Manual of Mental Disorders 4th Edition; WRAT = Wide Range Achievement Test.

All children in the ADHD group except one had previously been exposed to ADHD-related medication (dexamphetemine, catapres and/or methylphenidate). Half the children had been un-medicated for at least 1 month prior to testing. The eight children who were taking ADHD-related medication underwent a wash-out period of at least 24 hours prior to each test session.

The typically developing children (8 males; Mean age $=11$ years) had no previous diagnosis of ADHD, had current parent Global Index T-scores on the Conners' of $<60$, and no parent or teacher report of any learning disabilities or psychiatric/psychological problems. At the time of testing, children in the control group were administered a 2-subtest short-form of the Wechsler Abbreviated Scale of Intelligence, which reliably estimates full-scale IQ $(r=.93)$ (WASI, 1999). In addition, all children completed the WRAT Reading (WRAT-R) and Spelling (WRAT-S) subtests, and were required to score in the normative range for reading ability.

The two groups did not differ in terms of age, fullscale IQ, or reading ability (all $p>.05$ ) (Table 1 ). On all indices of the CPRS-R:L children with ADHD were reported as having significantly greater symptom severity than the control group (all subscales $p<.001$; see Table 1). All participants were right-handed, as assessed by the Edinburgh Handedness Inventory (Oldfield, 1971).

\section{Stimuli and procedure}

The paradigm used was a variant of the task described by Lavie and colleagues (2004, Experiment 3). The experimental task was run using E-prime software, and displayed on the 15-inch LCD screen of a PC laptop positioned at $60 \mathrm{~cm}$ directly in front of participants.

The flanker task display (see Figure 1) contained a target stimulus ' $x$ ' or ' $z$ ' $\left(.67^{\circ}\right.$ by $\left..48^{\circ}\right)$, lower case and italicised, that appeared equiprobably in one of six locations across a central horizontal row subtending $6.37^{\circ}$ from edge to edge. The target was accompanied in each display by a flanker (an uppercase ' $\mathrm{X}$ ' or ' $\mathrm{Z}$ ' subtending $1.05^{\circ} \times .57^{\circ}$ ) that appeared equiprobably above or below central fixation $\left(1.9^{\circ}\right)$. The flanker was equally likely to be congruent or incongruent with the target. In the low perceptual load conditions, targets appeared alone with the flanker. In the high perceptual load condition, the target appeared among five non-targets, (a)
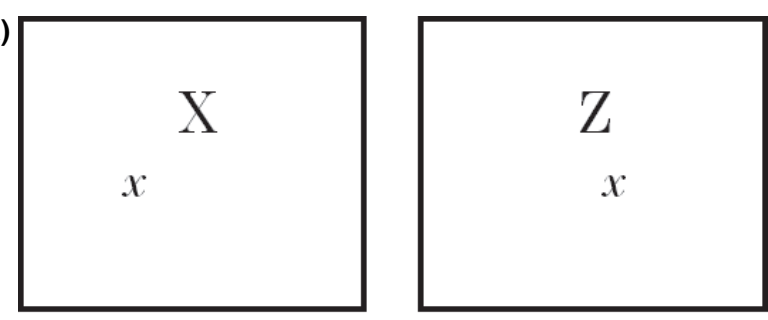

(b)
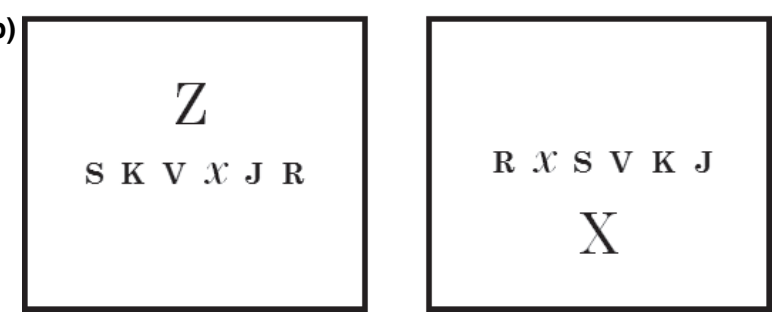

Figure 1 Schematic of the perceptual load manipulation on the flanker task. (a) Low perceptual load condition. (b) High perceptual load condition. In the examples the target letter is a lowercase ' $x$ ', and the flankers are an uppercase ' $\mathrm{X}$ ' or ' $\mathrm{Z}$ '

uppercase letters 'S', 'K', 'V', 'J', 'R' $\left(.67^{\circ} \times .48^{\circ}\right)$, which were randomly assigned to one of the five remaining locations across the central horizontal row. Participants were given $2000 \mathrm{~ms}$ to identify the target in each trial by pressing the corresponding button box (marked ' $x$ ' or ' $z$ ') using their left and right index finger, as quickly and accurately as possible. A $500 \mathrm{~ms}$ tone was given as feedback for an incorrect response or for not giving a response before the next trial began.

Participants performed 48 trials in each of the low and high perceptual load conditions across each of two testing sessions. Target and flanker identity and the position of targets (left, centre, right) were counterbalanced and randomised within each block.

\section{Results}

All children completed both behavioural testing sessions. However, there was significant variability in the data for Session 2 for both the ADHD and control group, thus weakening the main effects 
associated with the task conditions. ${ }^{1}$ For this reason, analyses focused on data from Session 1.

The mean reaction time (RT) and accuracy for responses to targets in the flanker task were analysed. Error trials were excluded from the RT analyses.

\section{Reaction time analyses}

To assess the effect of perceptual load and target position on distractor interference, a four-way, repeated-measures analysis of variance (ANOVA) with factors of perceptual load (low, high), target position (left, centre, right), congruency (congruent, incongruent) and group (control, ADHD) was performed. There was a significant main effect of perceptual load $\left[F(1,26)=16.93, p<.01, \eta^{2}=.394\right]$, with faster responses for low- $(\mathrm{M}=787 \mathrm{~ms})$ compared with highperceptual load trials ( $\mathrm{M}=888 \mathrm{~ms})$. A main effect of target position was also found $[F(1,26)=6.79$, $\left.p<.01, \eta^{2}=.207\right]$ which reflected faster responses for central $(M=806 \mathrm{~ms})$ compared with peripheral targets (Left: $M=858 \mathrm{~ms}$, Right: $M=849 \mathrm{~ms}$ ). A main effect of congruency $\left[F(1,26)=9.35, p<.01, \eta^{2}=.265\right]$ was driven by faster responses for congruent $(M=824 \mathrm{~ms})$ compared with incongruent trials ( $M=852 \mathrm{~ms})$. There was no main effect of group ( $p>.05)$.

Consistent with the load theory of selective attention, there was a significant interaction between perceptual load and congruency $[F(1,26)=7.34, p<.05$, $\left.\eta^{2}=.220\right]$, such that the difference in RT between congruent and incongruent trials (i.e., the interference effect(I.E.)) was significant under low perceptual load (Mean I.E. $=52 \mathrm{~ms} ; p<.01$, Bonferroni adjusted) but not under high perceptual load (Mean I.E. $=4 \mathrm{~ms}$; $p>.05$, Bonferroni adjusted) (see Figure 2). No significant interaction was found between perceptual load, congruency and group ( $p>$.05).

Crucially, the ANOVA revealed a significant threeway interaction between target position, congruency and group $\left[F(2,52)=3.20, p<.05, \eta^{2}=.110\right]$ that was modulated by a higher order four-way interaction that included perceptual load $[F(2,52)=6.19$, $\left.p<.01, \eta^{2}=.192\right]$.

Further analyses were conducted for each level of perceptual load separately. For the low perceptual load condition there was an interaction between target position, congruency and group $[F(2,52)$ $\left.=9.65, p<.01, \eta^{2}=.271\right]$. This interaction was not present under high perceptual load conditions

\footnotetext{
${ }^{1}$ Preliminary analysis on the session 2 data alone revealed the predicted main effect of perceptual load. However, the crucial main effect of congruency was not significant $[F(1,26)=.02$, $p>.05]$ and nor was the predicted interaction between perceptual load and congruency $[F(1,26)=1.87, p>.05]$. In contrast this predicted interaction was robustly found for the data from session 1 . The absence of these significant task effects in session 2 likely reflects more variable performance owing to decreased motivation in both children with ADHD and typically developing children to perform the task appropriately in the second session.
}

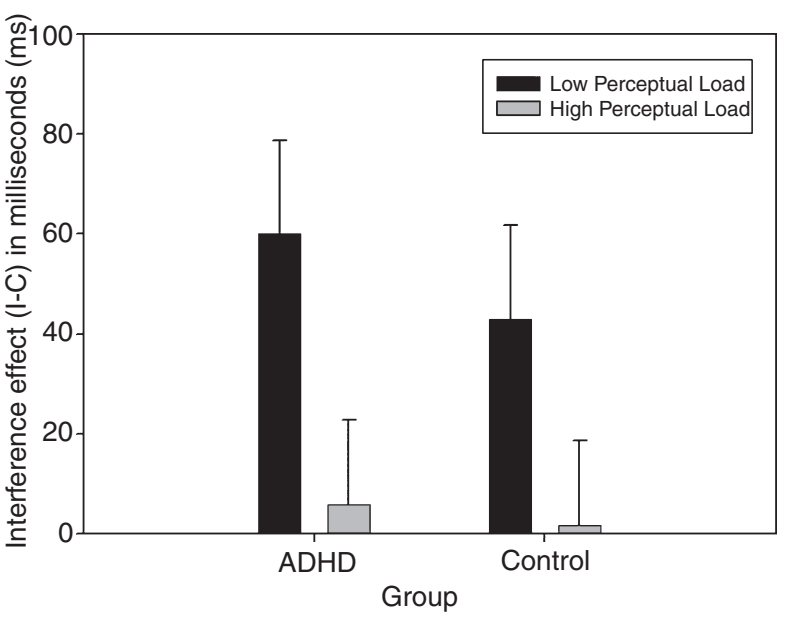

Figure 2 The effect of perceptual load on distractor interference expressed in terms of interference effect (Mean $\mathrm{RT}_{\text {Incongruent flankers }}-\mathrm{Mean} \mathrm{RT}_{\text {Congruent flankers }}$ in ms ( \pm 1 s.e.) for the ADHD and control group. A significant interference effect is found under low but not high perceptual load for both groups, as predicted by Lavie's Load theory of attention

$(p>.05)$ (see Figure 3). Pair-wise comparisons were performed for each group separately to assess whether the interference effect was significant for each of the target positions under the low perceptual load condition. Results revealed that RTs for incongruent, relative to congruent, trials were significantly slower in children with ADHD for right-sided targets (Mean I.E. $=147 \mathrm{~ms}, \quad$ S.E. $=41 ; \quad p<.01, \quad$ Bonferroni adjusted), but not for centre or left-sided targets $(p>$.05). Conversely, the control group showed significantly slower RTs for incongruent versus congruent trials for left-sided targets (Mean I.E. $=103 \mathrm{~ms}, \quad$ S.E. $=36 ; \quad p<.01, \quad$ Bonferroni adjusted), but not for centre or right-sided targets $(p>$.05). Thus, as hypothesised, children with ADHD showed increased interference from distractors when targets were presented on the right under low perceptual load, but reduced interference when presented centrally or to the left. In contrast, the typically developing children showed the opposite pattern of results, with increased interference effects when targets appeared on the left but not when presented centrally or on the right. The difference in interference effects between the ADHD and control group for left- and right-sided targets were significant (left: $t(26)=2.76, p<.05$; right: $t(26)=2.60$, $p<.05)$. These differences were associated with large Cohen's $d$ effect sizes of 1.04 and 1.26 , respectively. Comparison of the centrally presented targets, however, showed no significant difference between the two groups $(p>.05)$.

\section{Accuracy}

A similar four-way repeated measures ANOVA was performed for the accuracy data. Results revealed significant main effects of perceptual load $[F(1$, 

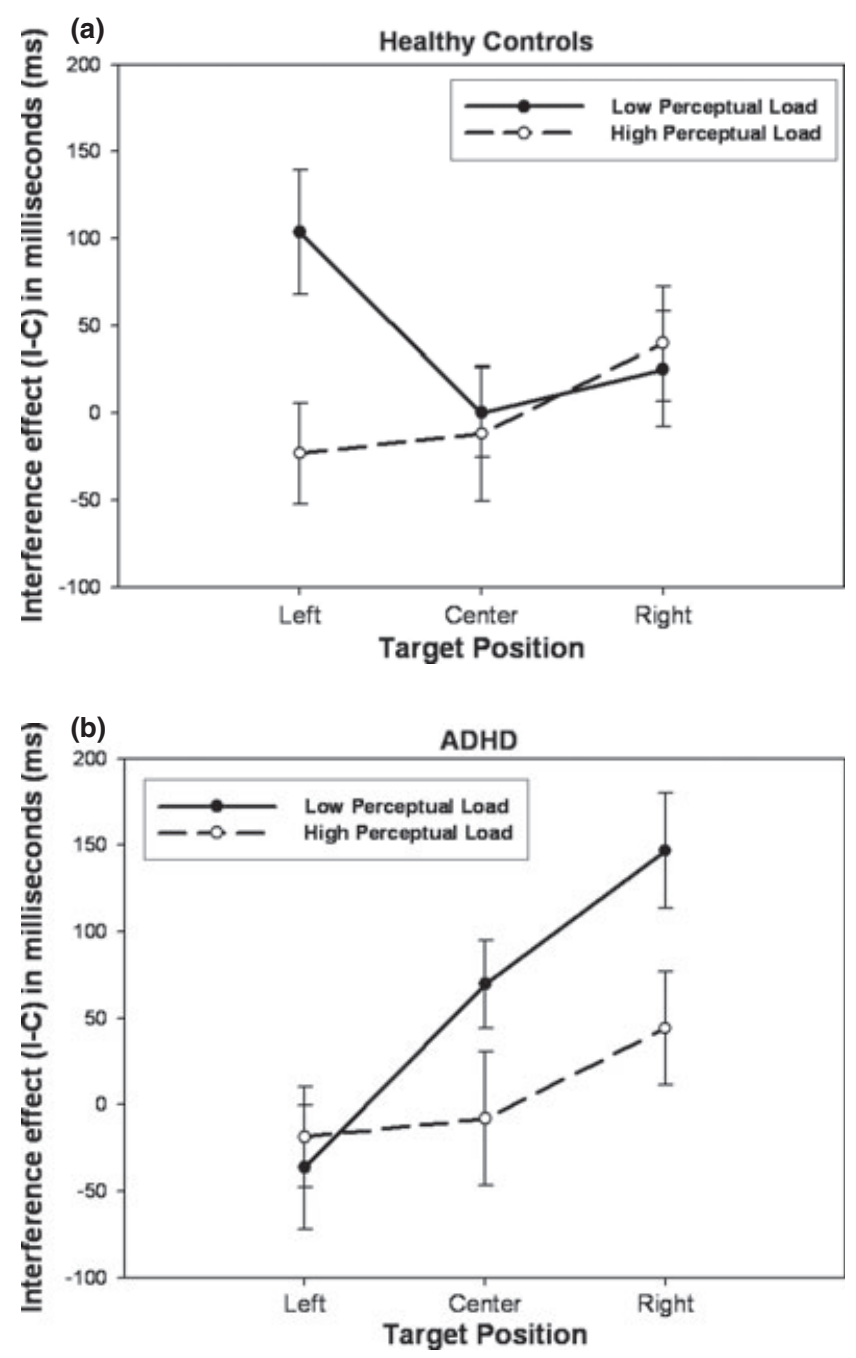

Figure 3 The mean interference effect in $\mathrm{ms}$ ( \pm 1 s.e.) for the perceptual load conditions as a function of target position for the (a) control group and (b) ADHD group

26) $\left.=16.91, p<.01, \eta^{2}=.394\right]$ and group $[F(1$, 26) $\left.=8.67, p<.01, \eta^{2}=.250\right]$, which reflected greater accuracy for low- $(M=86 \%)$ compared with highperceptual load ( $M=78 \%$ ), and greater overall accuracy in the control group $(M=87 \%)$ compared with the ADHD group ( $M=76 \%)$. There was also a significant interaction between perceptual load, target position and congruency $\left[F(2,52)=4.35, p<.05, \eta^{2}=.143\right]$. Separate pair-wise comparisons of the accuracy data as a function of target position for the low and high perceptual load conditions showed that participants were more accurate on congruent trials than incongruent trials only for right-sided targets under the high perceptual load condition (Mean difference $=8 \%$; $p<.05$, Bonferroni adjusted). No other significant main effects or interactions were found in the omnibus ANOVA regarding performance accuracy.

\section{Relationship between asymmetrical interference effects and ADHD symptomatology}

Additional analyses were performed to examine the relationship between the interference effect scores
(Mean $\mathrm{RT}_{\text {INCONGRUENT TRIALS }}$ - Mean RT $\mathrm{R}_{\text {CONGRUENT TRI- }}$ $A L S)$ and dimensional measures of ADHD symptomatology, as measured by the CPRS-R:L for both the ADHD and control group combined. A Spearman's correlation revealed a significant positive relationship between interference effect scores for rightsided targets under low perceptual load and DSM-IV Inattentiveness $(r=.392, p<.05)$ and Total symptom scores $(r=.392, p<.05)$. In contrast, there was a negative relationship between the interference effect for left-sided targets under low-perceptual load and DSM-IV Inattentiveness $(r=-.412, p<.05)$ and Total symptom scores $(r=-.455, p<.05)$. These correlations with dimensional measures of ADHD symptomatology are consistent with the findings from the ANOVA using a categorical diagnosis. Higher levels of ADHD symptomatology were therefore associated with increased interference when targets appeared on the right (as was seen in the ADHD group), whereas lower symptom levels were associated with increased interference when targets appeared on the left (as was seen in the control group).

\section{Re-analysis of data from Huang-Pollock et al. (2005)}

Confirmation of the spatial dependency of the reaction time effects reported above was sought via a re-analysis of the data from 76 participants (ADHD-I: 15; ADHD-CT: 27; controls: 34) presented in HuangPollock et al. (2005; refer to page 1215 for further details). There was a significant difference in the IQ estimates but not age between children with ADHD (irrespective of subtype) and the typically developing children [IQ: $F(1,74)=5.93, p=.017$; age: $F(1$, $74)=.007, p>$.05]. Parent- and teacher-rated Conners' Global Index T-scores were available for 70 of the participants.

Huang-Pollock et al. (2005) employed a selective attention paradigm that was similar in nature to that employed in the current study, differing, however, in the positioning of the stimulus. In their study, the target stimulus (X or $\mathrm{N}$ ) was positioned equiprobably around a circular array that had its centre at fixation, while the position of the distractor stimulus (either incongruent or neutral to the target) was lateralised, appearing either to the left or the right of the central stimulus array. The perceptual load of the array was manipulated by having the target appear alone (Set Size 1) or grouped with 1 (Set Size 2), 3 (Set Size 4) or 5 (Set Size 6) non-target letters ( $\mathrm{Z}, \mathrm{K}, \mathrm{Y}, \mathrm{V}$ or $\mathrm{H})$ positioned randomly around the array (see Figure 1, Huang-Pollock et al., 2005). For the current study, the spatial position of the distractor (left vs. right) was considered as a factor for analysis.

Reaction time analyses. Reaction times were submitted to a four-way, repeated-measures analysis of variance (ANOVA) with factors of distractor side (left, 
right), congruency (neutral, incongruent), perceptual load/set-size $(1,2,4,6)$ and group (control, ADHD). Since the children with ADHD and typically developing children differed significantly in terms of IQ, IQ was used as a covariate. Similar to the effects of target position in our study, increased interference effects for right-sided distractors under low, compared with high, perceptual load conditions in children with ADHD but not typically developing children was predicted.

There was a higher-order interaction between group, distractor side, congruency and set-size $[F(3,219)=2.65, p=.05]$. Interference effect scores (Mean RT $\mathrm{R}_{\text {INCONGRUENT TRIALS }}$ - Mean $\mathrm{RT}_{\text {NEUTRAL TRIALS) }}$ were derived and submitted to a group by distractor side ANOVA at each level of set-size. The interaction between group and distractor side was significant at set-size $2\left[F(1,73)=5.92, \quad p<.05, \quad \eta^{2}=.08\right]$ (Figure 4). Analysis of simple main effects with Bonferroni adjustments revealed that control children displayed higher interference effects when distractors appeared on the left relative to the right $(p=.024)$. Further, as predicted, children with ADHD displayed higher interference effects when distractors appeared on the right $(p<.05$; Cohen's $d=.5)$ but not on the left $(p>.05)$, relative to the typically developing children. The interaction between group and distractor side was not significant at any other set-size.

Relationship between asymmetrical interference effects and ADHD symptomatology. Again the relationship between interference effect scores and dimensional measures of ADHD symptomatology, as measured by the Conners' scales, was examined for both the ADHD and control group combined. A partial correlation, controlling for IQ differences, revealed a significant positive relationship between interference effect scores for right-sided distractors

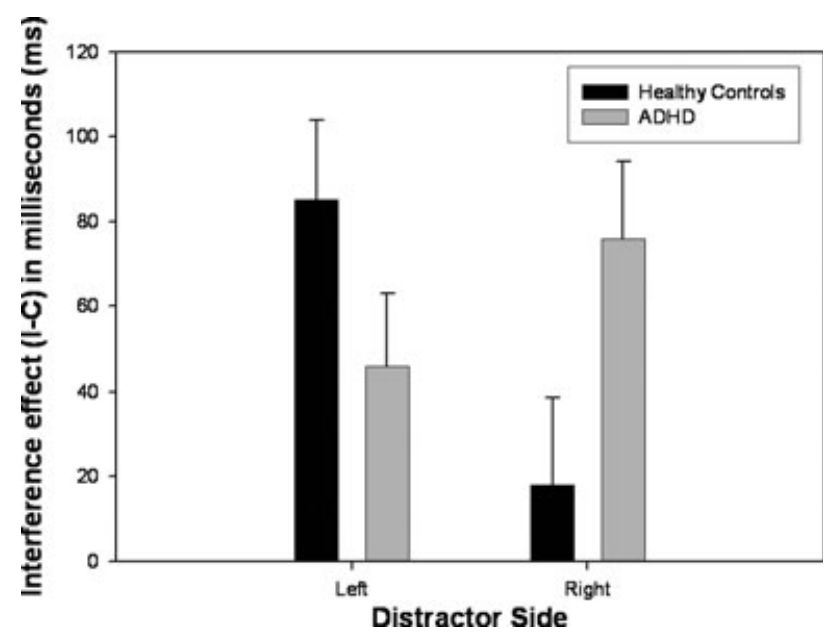

Figure 4 The mean interference effect in $m s$ (+1 s.e.) as a function of distractor position for children with ADHD and controls (re-analysis of data presented in HuangPollock et al., 2005) at set size 2 (the source of the higher-order interaction) and parent-rated Conners' Global Index T-scores $(r=.27, p=.013$, one-tailed) and Cognitive Problems T-score $(r=.28, p=.011$, one-tailed). In contrast, there was a negative relationship between the interference effect for left-sided distractors at setsize 2 and Conners' Global Index T-scores $(r=-.21$, $p=.04$, one-tailed). Thus in both samples higher levels of parent-rated ADHD symptoms were associated with increased right-sided interference effects under low perceptual load, whereas lower symptom levels were associated with increased left-sided interference effects.

\section{Discussion}

The existence of selective attention deficits in children with ADHD is debated within the literature. The current study examined whether manipulations of perceptual load and the spatial location of the targets could unmask a subtle abnormality of selective attention in ADHD. Robust differences between the groups emerged as a function of the spatial location of the targets. Children with ADHD displayed pronounced interference effects when targets appeared on the right, and diminished interference effects when targets appeared on the left, under low- but not high-perceptual load. Typically developing children, on the other hand, showed the opposite pattern of interference effects, with greater interference for leftversus right-sided target displays. Moreover, greater interference for right-sided target displays under low perceptual load was associated with greater ADHD-symptom severity. These findings were also confirmed in a re-analysis of an independent and previously published data set based upon a selective attention task similar to the one described in the current paper (Huang-Pollock et al., 2005).

The findings from this study are consistent with those of Huang-Pollock et al. (2005), who found that children with ADHD were not manifestly impaired on tasks of selective attention. Our results also accord with a number of studies that have failed to document significant impairments when measuring selective attention using variants of the Stroop, Eriksen flanker and Posner cuing tasks (Brodeur \& Pond, 2001; Huang-Pollock \& Nigg, 2003; HuangPollock et al., 2005; Sergeant et al., 2003). In line with Lavie's load theory of attention (1995), distractor interference effects were increased under low perceptual load conditions and attenuated under high perceptual load conditions, in both children with ADHD and in typically developing children. If children with ADHD suffered from a generalised (i.e., non-spatial) restriction of attentional capacity then one would have expected greater attenuation of distractor effects under low perceptual load conditions, relative to that seen in typically developing children. That this did not occur argues against a general 
deficit of selective attention in children with ADHD in the current study.

Our results do suggest, however, the existence of abnormal spatial asymmetries in ADHD. Acquired damage to the right cerebral hemisphere in adults can lead to the syndrome of unilateral spatial neglect in which there is an asymmetry (or bias) of spatial attention toward the ipsilesional side (Driver $\&$ Mattingley, 1998). This spatial bias can give rise to reduced awareness for contralesional stimuli, and to hyper-distractibility for ipsilesional distractors (Lavie \& Robertson, 2001). The results of the current study suggest that children with ADHD display a spatially distinct pattern of distractor interference under low perceptual load, compared with typically developing children. Children with ADHD displayed more interference when targets appeared on the right under low perceptual load, and less interference when targets appeared on the left. For a number of reasons, these results are consistent with a rightward spatial bias (left spatial inattention) in children with ADHD. First, if attentional resources are directed to right space, then processing of distractor information at this location should be facilitated. Second, if attentional resources are directed away from left space (left inattention) then there should be reduced attentional capacity to process distractor information at leftward spatial locations.

Geeraerts et al. (2007) recently examined the ability of adults with ADHD to ignore a task-irrelevant distractor stimulus that was presented in the opposite hemi-field to a target. Using psychophysical procedures, Geeraerts et al. showed that adults with ADHD were poorer at detecting targets in the left hemi-field when they were accompanied by a distractor stimulus in the right hemi-field. Comparable results were also seen in patients with right parietal lesions (Geeraerts, Lafosse, Vandenbussche, \& Verfaillie, 2005). Healthy adult participants showed no such asymmetry. It seems plausible, therefore, that the neural substrate of increased right-sided interference effects could be dysfunction within right-hemisphere attention networks including the parietal lobe. Our results are also consistent however with theories which posit that neglect-like behaviour in ADHD arises from asymmetrical frontostriatal function (Voeller \& Heilman, 1988; Heilman et al., 1991).

Given the specific nature of our findings, we sought confirmation of the effects in an independent cohort. Huang-Pollock et al. (2005) had previously employed a selective attention task in which distractors were positioned to the left or right of a central, circular stimulus array. These authors did not, however, analyse distractor position as an independent factor. In line with our hypothesis, including this factor in the re-analysis yielded evidence for increased right-sided interference effects in children with ADHD at low (set-size 2) but not high perceptual loads (set-size 4 or set-size 6). It should be noted that the failure to find an effect of distractor location under the lowest perceptual load condition (set-size 1; distractor appeared alone with the target) stands as a limitation of the current study. Nevertheless, the similarity between the results reported across two separate cohorts using subtly different methodologies supports the robustness of our findings.

Interestingly, the typically developing children also displayed a spatially asymmetric interference effect pattern under low load, but in the opposite direction to children with ADHD (see Figures 3 and 4). Across both studies the typically developing children showed increased interference effects for left-sided stimuli and reduced interference effects for rightsided stimuli. This pattern is consistent with observations of a leftward spatial bias in healthy participants, a phenomenon known as pseudoneglect because it mirrors the spatial biases displayed by patients with the neglect syndrome (Bowers \& Heilman, 1980; McCourt \& Jewell, 1999; Nicholls, Mattingley, \& Bradshaw, 2005). The leftward spatial bias in neurological healthy participants is thought to arise because of asymmetric activation of the hemispheres, with spatial tasks activating the right-hemisphere more than the left-, thus biasing attention leftward (Kinsbourne, 1970). Alternatively, pseudoneglect may reflect asymmetries in the neural substrates that control attention (Heilman et al., 1987; Nicholls \& Roberts, 2002). In the current context, a leftward attentional bias would render typically developing children more prone to interference on the left, but not right. This effect likely arises because residual attentional resources are available on the left under low perceptual load to process the distractors.

One potential limitation of the current study is that both cohorts of children with ADHD had high rates of comorbid disorders, including conduct disorder and oppositional defiant disorder. It is therefore possible that our findings of increased right-sided interference effects are not specific to ADHD per se. Nevertheless, it seems likely that the relationship between asymmetric attentional performance and ADHD symptom ratings should generalise to other clinic-referred ADHD samples where similar rates of comorbidity are reported.

Although the current study was based upon a relatively small sample of 28 well-matched participants, a noteworthy aspect is the robust effect size (e.g., Cohen's $d=1.26$ ) associated with distractor effects for right-sided targets. Effect sizes of this magnitude are rare in the selective attention literature of ADHD. The analogous comparison in the data of HuangPollock et al. yielded an effect size of .50, although it should be noted that IQ differences between the groups may have somewhat reduced the effect size. Furthermore, right-sided interference effects were positively correlated with ADHD symptom scores in 
both cohorts. By contrast, left-sided interference effects were negatively correlated with symptom scores. Thus the categorical distinctions between increased right-sided interference effects in children with ADHD and increased left-sided interference effects in the typically developing children were also reflected in dimensional ratings of ADHD behaviour. At this stage the functional impact of abnormal spatial asymmetries in ADHD remains unclear. Future studies should assess the relationship between asymmetries of visual attention and functional impairment at school and home. Conceivably, abnormalities in the allocation of visual attention in space could impact upon academic abilities such as reading in children with $\mathrm{ADHD}$.

Although it should be noted that generalised deficits of selective attention may be documented in ADHD using larger samples and alternative paradigms (see Johnson et al., 2008), our results suggest that future assessments of selective attention in ADHD should take into account the spatially dependent nature of performance.

\section{Acknowledgements}

This work was supported by a Howard Florey Centenary Fellowship to M.A.B from the National Health and Medical Research Council (NHMRC) of Australia. Data collection at the Royal Children's Hospital Melbourne was supported in part by a grant from the Murdoch Children's Research Institute. The authors would like to acknowledge the assistance of Thomas Carr and Joel Nigg and the Michigan State project. The data collection associated with the supplementary analysis of Huang-Pollock et al. (2005) was supported in part by ROI MH59105 to Joel Nigg and MH12333 to Cynthia Huang-Pollock. The authors would like to thank the participating families for their involvement.

\section{Correspondence to}

Edgar Chan, The University of Queensland, School of Psychology and Queensland Institute, Brisbane, Australia; Email: echan@psy.uq.edu.au

\section{Key points}

- Despite clinical evidence of attentional deficits in ADHD, findings from classic selective attention paradigms have proved inconclusive.

- The current study examined whether the interference engendered by a flanking stimulus varied as a function of perceptual load and the spatial location of targets.

- Across two independent cohorts, children with ADHD displayed greater interference effects on the right, but not left side under low perceptual load - a pattern that was exactly opposite to typically developing children. Attentional asymmetry correlated with reported levels of ADHD symptomatology.

- These findings suggest an abnormal pattern of attentional asymmetry in children with ADHD that may reflect disturbance to right-hemisphere attentional networks.

\section{References}

American Psychiatric Association. (1994). Diagnostic and statistical manual of mental disorders (4th edn) [DSM-IV]. Washington, DC: Author.

Booth, J.R., Burman, D.D., Meyer, J.R., Lei, Z., Trommer, B.L., Davenport, N.D., Li, W., Parrish, T.B., Gitelman, D.R., \& Mesulam, M.M. (2005). Larger deficits in brain networks for response inhibition than for visual selective attention in attention deficit hyperactivity disorder (ADHD). Journal of Child Psychology and Psychiatry, 46, 94-111.

Bowers, D., \& Heilman, K.M. (1980). Pseudoneglect: Effects of hemispace on a tactile line bisection task. Neuropsychologia, 18, 491-498.

Broadbent, D.E. (1958). Perception and communication. Oxford: Oxford University Press.

Brodeur, D.A., \& Pond, M. (2001). The development of selective attention in children with ADHD. Journal of Abnormal Child Psychology, 29, 229-239.

Conners, C.K. (1997). Conners' Rating Scales-Revised: Technical manual. Toronto: Multi-Health Systems.

Driver, J., \& Mattingley, J.B. (1998). Parietal neglect and visual awareness. Nature Neuroscience, 1, 17-22.
Duncan, J. (1980). The locus of interference in the perception of simultaneous stimuli. Psychological Review, 87, 272-300.

Eriksen, B.A., \& Eriksen, C.W. (1974). Effects of noise letters upon the identification of a target letter in a nonsearch task. Perception and Psychophysics, 16, 143-149.

Geeraerts, S., Lafosse, C., Vaes, N., Vandenbussche, E., $\&$ Verfaillie, K. (2007). Dysfunction of right-hemisphere attentional networks in attention deficit hyperactivity disorder. Journal of Clinical and Experimental Neuropsychology, 12, 1-11.

Geeraerts, S., Lafosse, C., Vandenbussche, E., \& Verfaillie, K. (2005). A psychophysical study of visual extinction: Ipsilesional distractor interference with contralesional orientation thresholds in visual hemineglect patients. Neuropsychologia, 43, 530-541.

Heilman, K.M., Bowers, D., Valenstein, E., \& Watson, R.T. (1987). Hemispace and hemispatial neglect. In M. Jeannerod (Ed.), Neurophysiological and neuropsychological aspects of spatial neglect (pp. 115-150). Amsterdam: Elsevier.

Heilman, K.M., Voeller, K.K., \& Nadeau, S.E. (1991). A possible pathophysiologic substrate of attention 
deficit hyperactivity disorder. Journal of Child Neurology, 6, S76-81.

Huang-Pollock, C.L., \& Nigg, J.T. (2003). Searching for the attention deficit in attention deficit hyperactivity disorder: The case of visuospatial orienting. Clinical Psychology Review, 23, 801-830.

Huang-Pollock, C.L., Nigg, J.T., \& Carr, T.H. (2005). Deficient attention is hard to find: Applying the perceptual load model of selective attention to attention deficit hyperactivity disorder subtypes. Journal of Child Psychology and Psychiatry, 46, 1211-1218.

Johnson, K.A., Robertson, I.H., Barry, E., Mulligan, A., Dáibhis, A., Daly, M., et al. (2008). Impaired conflict resolution and alerting in children with ADHD: Evidence from the Attention Network Task (ANT). Journal of Child Psychology and Psychiatry, 49, 1339-1347.

Kinsbourne, M. (1970). The cerebral basis of lateral asymmetries in attention. Acta Psychologica, 33, 193201.

Lavie, N. (1995). Perceptual load as a necessary condition for selective attention. Journal of Experimental Psychology: Human Perception and Performance, 21, 451-468.

Lavie, N., Hirst, A., de Fockert, J.W., \& Viding, E. (2004). Load theory of selective attention and cognitive control. Journal of Experimental Psychology: General, 133, 339-354.

Lavie, N., \& Robertson, I. H. (2001). The role of perceptual load in neglect: Rejection of ipsilesional distractors is facilitated with higher central load. Journal of Cognitive Neuroscience, 13, 867-876.

McCourt, M.E., \& Jewell, G. (1999). Visuospatial attention in line bisection: Stimulus modulation of pseudoneglect. Neuropsychologia, 37, 843-855.

Nicholls, M.E., Mattingley, J.B., \& Bradshaw, J.L. (2005). The effect of strategy on pseudoneglect for luminance judgements. Brain Research. Cognitive Brain Research, 25, 71-77.
Nicholls, M.E., \& Roberts, G.R. (2002). Can free viewing perceptual asymmetries be explained by scanning, pre-motor or attentional bias? Cortex, 38, 113-136.

Oldfield, R.C. (1971). The assessment and analysis of handedness: The Edinburgh inventory. Neuropsychologia, 9, 97-113.

Polanczyk, G., \& Rohde, L.A. (2007). Epidemiology of attention-deficit/hyperactivity disorder across the lifespan. Current Opinion in Psychiatry, 20, 386-392.

Sergeant, J.A., Geurts, H., Huijbregts, S., Scheres, A., \& Oosterlaan, J. (2003). The top and the bottom of ADHD: A neuropsychological perspective. Neuroscience and Biobehavioral Reviews, 27, 583-592.

Sheppard, D.M., Bradshaw, J.L., Mattingley, J.B., \& Leeb, P. (1999). Effects of stimulant medication on the lateralisation of line bisection judgements of children with attention deficit hyperactivity disorder. Journal of Neurology, Neurosurgery, and Psychiatry, $66,57-63$

Sowell, E.R., Thompson, P.M., Welcome, S.E., Henkenius, A.L., Toga, A.W., \& Peterson, B.S. (2003). Cortical abnormalities in children and adolescents with attention-deficit hyperactivity disorder. Lancet, 362, 1699-1707.

Vance, A., Silk, T.J., Casey, M., Rinehart, N.J., Bradshaw, J.L., Bellgrove, M.A., et al. (2007). Right parietal dysfunction in children with attention deficit hyperactivity disorder, combined type: A functional MRI study. Molecular Psychiatry, 12, 826-832.

Voeller, K.K., \& Heilman, K.M. (1988). Attention deficit disorder in children: A neglect syndrome? Neurology, 38, 806-808.

WASI. (1999). Wechsler Abbreviated Scale of Intelligence Manual. New York: The Psychological Corporation.

Manuscript accepted 20 January 2009 